Sphingosine kinase 2 in autoimmune/inflammatory disease and development of sphingosine kinase 2 inhibitors

$$
\text { Nigel J Pyne }{ }^{\mathrm{a} *} \text {, David R. Adams }{ }^{\mathrm{b}} \text { and Susan Pyne }{ }^{\mathrm{a}}
$$

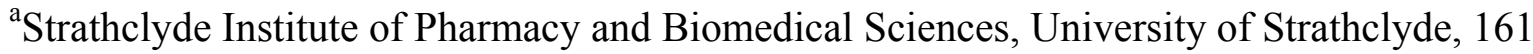

Cathedral St, Glasgow, G4 0RE, Scotland, UK; ${ }^{\mathrm{b}}$ Institute of Chemical Sciences, Heriot-Watt University, Edinburgh, EH14 4AS, Scotland, UK.

* To whom correspondence should be addressed (email: n.j.pyne@strath.ac.uk)

Key words: Sphingosine kinase, auto-immune disease, interleukin, interferon- $\gamma$, histone deacetylase, related orphan receptor- $\gamma \mathrm{t}$

Short title: Sphingosine kinase 2 and autoimmune disease 
Abstract-The purpose of this opinion is to present a case for targeting sphingosine kinase 2 (SK2) in autoimmune/inflammatory disease. Data obtained using Sphk2 ${ }^{-/-}$mice suggests that SK2 is an anti-inflammatory enzyme, although this might be misleading because of a compensatory increase in expression of a second isoform, sphingosine kinase 1 (SK1), which functions as a pro-inflammatory enzyme. SK2 is involved in regulating interleukin12/interferon- $\gamma$ and histone deacetylase-1/2 signalling and potentially retinoid related orphan receptor- $\gamma$ t stability linked with Th17 cell polarisation. Therefore, there is a need to develop highly potent SK2 inhibitors with selectivity over SK1 to clearly define the role of SK2 in autoimmune/inflammatory disease. Structural determinants of SK2 relative to SK1 will enable design of selective SK2 inhibitors.

\section{Sphingosine 1-phosphate Signalling}

The bioactive lipid, sphingosine 1-phosphate (S1P) is formed by the phosphorylation of sphingosine and this reaction is catalysed by two isoforms of sphingosine kinase, termed SK1 and SK2. These enzymes are encoded by different genes and are localised in distinct subcellular compartments. The enzymes also exhibit distinct biochemical properties and inhibitor sensitivities [1]. S1P is degraded by S1P lyase to produce (E)-2-hexadecenal and phosphoethanolamine and is dephosphorylated by S1P phosphatase to reform sphingosine [1]. S1P is released from cells using specific transporters (e.g. Spns2) (see Glossary) in the plasma membrane and binds to a family of G protein-coupled receptors (GPCR), the S1P receptors $\left(\mathrm{S} 1 \mathrm{P}_{1}-\mathrm{S}_{\mathrm{P}}\right)[2,3]$ on cells to induce biological responses. Intracellular S1P formed by SK2 can bind to various targets, such as histone deacetylase 1/2 (HDAC-1/2) [4], human telomerase reverse transcriptase (hTERT) [5] and prohibitin 2 [6], thereby linking the enzyme with epigenetic regulation, aging and mitochondrial respiratory complex assembly respectively. 
SK1 and SK2 exhibit some redundancy in function, as evident from studies in Sphk $1^{-/-}$or Sphk $2^{-/-}$mice, which are phenotypically healthy while deletion of both genes is embryonic lethal [7]. These enzymes appear to regulate discrete sub-cellular pools of intracellular S1P that govern both overlapping and/or distinct biology. The importance of SK2 is evinced by the fact that it has a critical role in governing the clinical efficacy of FTY720 (Gilenya ${ }^{\circledR}$ ), which is phosphorylated by SK2 to produce FTY720-phosphate and which functions to modulate S1P receptors. SK1 and SK2 are regulated by post-translational modification and interact with specific proteins and lipids (for review see [8]). For instance, both enzymes are phosphorylated by extracellular signal-regulated kinase (ERK) and this promotes translocation of SK1 from the cytoplasm to the plasma-membrane, where it can access sphingosine $[9,10]$. In contrast, SK2 is localised to the endoplasmic reticulum, nucleus or is associated with mitochondria [11]. In addition, SK2 contains nuclear localisation and export sequences and when phosphorylated by protein kinase D is exported from the nucleus to the cytoplasm [12]. The aim of this opinion is to evaluate the role of SK2 in autoimmune/inflammatory disease. This is both necessary and timely because data obtained using $\mathrm{Spk}^{-/-}$mice suggest an anti-inflammatory role for this enzyme, while SK2 inhibitors indicate a pro-inflammatory role. It is important to appraise this issue so that the full potential of SK2 as a target for therapeutic intervention in autoimmune/inflammatory disease can be evaluated. We have therefore discussed the data obtained with Sphk2 $2^{-/-}$mice, assessed the role of SK2 in IL-12 signalling and Th17 polarisation that contribute to autoimmune disease and have provided information on how inhibitor selectivity for SK2 over SK1 inhibitors can be achieved. This is necessary to distinguish SK2 function from that of SK1 and to provide impetus for the use of SK2 inhibitors in autoimmune/inflammatory disease. 


\section{Consideration of evidence obtained with Sphk2 ${ }^{-/-}$mice}

Data obtained using $\mathrm{Sphk} 2^{-/}$mice suggest that SK2 might function as an antiinflammatory enzyme. In our opinion, the Sphk $2^{-/-}$model is not entirely informative as genetic knockout or RNAi knockdown of SK2 in cells can lead to increased expression of SK1 [13-15]. In addition, Sphk $2^{-/-}$mice exhibit higher S1P levels in whole blood, serum, lymphatic fluid and mesenteric lymph nodes and it has been suggested that this is due to a compensatory increase in SK1 expression [16]. Other studies have shown no change in SK1 mRNA in brain, heart and lung, liver, spleen and kidney in Sphk $2^{-/-}$mice, although protein expression was not investigated [7]. Clearly, further studies are required to investigate the inter-relationship between SK2 and SK1. Nevertheless, the pro-inflammatory effects observed in Sphk2 $2^{-/-}$mice might be attributable to increased SK1 expression. Indeed, Sphk $1^{-/-}$mice are protected from ulcerative colitis, indicating that SK1 functions as a pro-inflammatory enzyme in this disease [17]. Such an increase in SK1 expression levels in the Sphk2 $2^{-/-}$mice is not recapitulated with selective SK2 inhibitors. These data indicate that there is a fundamental difference in how chronic loss of SK2 protein through genetic knockout/knockdown versus inhibition of SK2 activity with small molecules can affect SK1 expression. In our opinion, this has hindered evaluation as to whether SK2 is, in fact, a pro-inflammatory enzyme and therefore, a viable target for intervention in autoimmune/inflammatory disease. In addition, Kharel et al. [18] observed that the treatment of mice with the SK2 inhibitor, SRL080811 increased blood S1P levels. This contrasts with the effect of SK2 inhibitor, ABC294640 which lowers S1P levels [19]. Since SRL080811 recapitulated the effect of Sphk2 $2^{-/-}$mice, the authors have suggested that SK2 might exhibit additional activities in regulating S1P uptake into cells. However, the effects of SRL080811 and the Sphk2 knockout might represent mutually exclusive events. 
Information from the Sphk $2^{-/-}$mice needs to be very carefully de-convoluted. A couple of examples are given here. First, Samy et al. [20] demonstrated that Sphk2 ${ }^{-/-} \mathbf{C D}^{+}$cells exhibit a hyper-activated phenotype with significantly enhanced proliferation and cytokine secretion (IFN- $\gamma$ ) in response to IL-2, as well as reduced sensitivity to $\mathrm{T}(\mathrm{reg})$-mediated suppression in vitro. However, the authors suggested that these effects are independent of S1P and S1P receptors. The former is based on the finding that intracellular S1P levels are not altered in Sphk2 ${ }^{-/-} \mathrm{CD}^{+}$cells. This is, in fact, consistent with increased SK1 expression rebalancing S1P levels and/or possible non-catalytic functions of SK2 affecting $\mathrm{CD}^{+}$cells; the latter which would not be recapitulated by SK2 inhibitors. Therefore, the observed effects of Sphk2 knockout on IL-2 signalling in these cells might be mediated by SK1.

Second, Bajwa et al. [21] demonstrated that Sphk2 $2^{-/-}$mice do not develop kidney fibrosis in response to injury induced by folic acid and that this is associated with increased levels of the pro-inflammatory mediator, IFN- $\gamma$, which is anti-fibrotic. Thus, SK2 is suggested to be a negative regulator of IFN- $\gamma$. However, an earlier publication by this group demonstrated that SK1 mRNA transcript levels are increased in SK2 null mice in kidneys subjected to ischaemic/reperfusion injury [22]. Moreover, overexpression of SK1 increases IL-12 formation from dendritic cells and RNAi knockdown in these cells prevents IFN- $\gamma$ formation from Th1 cells [23]. Therefore, the effects on IFN- $\gamma$ formation in Sphk2 $2^{-/-}$mice might be attributable to increased expression of SK1. A significant finding in the study by Bajwa et al. [21] was the demonstration that the number of infiltrating $\mathbf{C D 3}^{+} \mathrm{T}$ cells, $\mathrm{CD} 11 \mathrm{~b}^{+}$neutrophils and macrophages in the fibrotic kidney from Sphk $2^{-/-}$mice are considerably reduced compared with WT mice. This cannot be explained by an increase in SK1 expression in Sphk2 $2^{-/}$mice, as this would potentially increase inflammatory cell infiltration in the kidney. Therefore, the antiinflammatory profile is likely attributable to the loss of SK2 and this supports our proposal that SK2 is, in fact, a pro-inflammatory enzyme that can affect T-cell dynamics. In addition, folic 
acid increased mRNA expression levels of innate pro-inflammatory mediators e.g. CXCL1 and CXCL2 in WT and Sphk1 $1^{-/-}$mice but not Sphk2/-- mice.

There is more direct evidence obtained using Sphk2 $2^{-/-}$mice that SK2 can function in a pro-inflammatory context as these mice are protected against experimental auto-immune encephalomyelitis (EAE) [24], a disease model of Multiple Sclerosis (MS). In addition, we have shown that the $\mu \mathrm{M}$ potent SK2 inhibitor, (R)-FTY720 methyl ether (ROMe) prevents inflammatory cell infiltration into the spinal cord and recapitulates the Sphk2 knockout in reducing disease progression in the EAE model [25]. In addition, the SK2 inhibitor, Yeliva ${ }^{\circledR}$ (ABC294640) exhibits anti-inflammatory activity in rodent models of Inflammatory Bowel Disease (IBD) [26-28] and arthritis [29]. Finally, hydroxy-based SK1/SK2 inhibitors have also been shown to exert anti-inflammatory activity in colitis in mice [30]. Therefore, there is some preliminary evidence that SK2 inhibitors might be exploited for the treatment of autoimmune/inflammatory disease.

\section{Rationale for targeting sphingosine kinase 2}

SK1 is involved in so-called 'inside-out' signalling, where S1P formed by SK1 is released from cells through transporters to act on S1P receptors in an autocrine manner [31]. Evidence that SK2 can perform a similar function is scant. However, SK2 catalyses the phosphorylation of FTY720, and the phosphorylated FTY720 can be released from cells to functionally antagonise $\mathrm{S}_{1} \mathrm{P}_{1}$ on T-cells [32]. Significantly, this underlies the mechanism by which FTY720 phosphate modulates T-cell polarisation and trafficking and which is clinically effective in MS patients. Therefore, SK2 can indirectly regulate S1P receptors and this is consistent with the partial redundancy of function seen in $\mathrm{Sphk} 1^{-/-}$or $\mathrm{Sphk} 2^{-/-}$mice. It follows that the selective inhibition of SK2 activity has the potential to block localized S1P synthesis, thereby reducing availability of S1P at S1P receptors and at intracellular targets (e.g. HDAC-1/2) 
involved in inflammatory disease-forming mechanisms (Figure. 1). This is a plausible therapeutic approach as S1P is involved in IBD, Psoriasis (Ps) and MS [33, 34].

\section{Target validation of SK2 in Th1 driven auto-immune disease}

Is there evidence that SK2 can function in molecular signalling pathways that affect $\mathrm{T}$ cell sub-sets that drive auto-immune disease? In this regard, the IL12 $\beta 1$ receptor, which binds IL-12, has been shown to form a complex with SK2 [35]. Moreover, over-expression of dominant negative or wild-type SK2 reduce and enhance IL-12-stimulated IFN- $\gamma$ formation respectively in T-cells (Figure 2) [35]. Therefore, it would be of considerable interest to evaluate the effect of more potent SK2 inhibitors with selectivity over SK1 and in combination with for example, IL-12 antibodies to establish whether there is improved efficacy in Th1-mediated disease models compared with either agent alone (Figure 3). In theory, decreasing IL-12 levels with antibody should reduce the extent to which SK2 can be activated by IL-12 and this might enable SK2 inhibitors to more effectively block IL-12-stimulated IFN- $\gamma$ production.

\section{SK2 and potential link with STAT3, IL-6 and IL-17}

SK2 might also play a significant role in Th17 biology. This is based on the fact that the IL-23 receptor dimerizes with IL12 $\beta 1$ receptor required for IL-23-stimulated IL-17 formation [36]. Indeed, there is substantial evidence supporting a role for $\mathrm{S} 1 \mathrm{P}$ in promoting Th17 polarisation [37]. For instance, S1P increases phosphorylated Signal Transducer Activator of Transcription 3 (STAT3) levels and IL-6 formation [38]; the latter is a major stimulator of Th17 polarisation. In addition, STAT3 increases ROR- $\gamma \mathbf{t}$ expression [39] and STAT3 or ROR- $\gamma \mathbf{t}$ knockout reduces Th17 polarisation [39, 40]. Based on these findings, it is possible that S1P stimulates Th17 polarisation via a ROR- $\gamma \mathrm{t}$-dependent mechanism. Therefore, one could evaluate the effect of more potent SK2 inhibitors with selectivity over SK1 in combination with IL-17 
antibodies to establish whether there is improved efficacy in Th17-mediated disease models compared with either agent alone (Figure 3). SK2 inhibitors are expected to reduce IL-17 formation, thereby increasing the capacity for IL-17 antibody (at a given dose) to neutralise the lower amounts of IL-17 produced.

\section{SK2 and potential link with T(reg) and auto-immune disease}

S1P also inhibits $\mathbf{T}(\mathbf{r e g})\left(\right.$ Foxp $\left.^{+}\right)$[41] and this involves an $\mathrm{S} \mathrm{P}_{1}$-dependent negative regulation of TGF $\beta$ signalling that requires SK activity and is blocked by SK inhibitors. Both SK1 and SK2 in T(reg) are up-regulated by TGF $\beta$ [41]. However, SK1 normally promotes TGF $\beta$ signalling. For instance, SK1 is involved in TGF $\beta$-stimulated fibroblast transformation

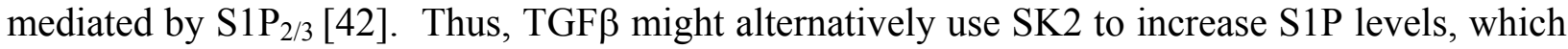
via 'inside-out' signalling acts on $\mathrm{S}_{1} \mathrm{P}_{1}$, thereby inhibiting $\mathrm{TGF} \beta$-induced $\mathrm{T}(\mathrm{reg})$ formation in a negative feedback manner. Thus, SK2 inhibitors might enhance $\mathrm{T}(\mathrm{reg})$ function to suppress autoimmune disease and this could be potentially augmented by combination with anti-IL-12 and IL-17 antibodies.

\section{SK2 and potential link with ROR- $\gamma \mathrm{t}$}

HDAC-1 catalyses de-acetylation of ROR- $\gamma \mathrm{t}$ [43] and reduces its stability in cells. Indeed, HDAC1 inhibitors maintain acetylated ROR- $\gamma$ t to increase Th17 polarisation [43]. Significantly, S1P formed by SK2 inhibits HDAC1 [44]. Thus, SK2 inhibitors, in reducing S1P levels might reactivate $\mathrm{HDAC1}$ and promote ROR- $\gamma$ t deacetylation and degradation, thereby limiting Th17 polarisation. This also provides a rationale for evaluating whether combined treatment with SK2 inhibitors and ROR- $\gamma$ t inverse agonists improves efficacy in Th17-mediated disease models (Figure. 3). In reducing ROR- $\gamma$ t stability, SK2 inhibitors might enable inverse agonists to decrease ROR- $\gamma$ t activity to below threshold levels required to promote IL-17 
formation. Such threshold levels are likely to exist as weak strength T-cell activation is sufficient to stimulate IL-17 formation [45].

\section{Potential advantages of targeting SK2 over S1P receptors in auto-immune disease}

A major effort in tackling auto-immune disease involves the use of $\mathrm{S}_{1} \mathrm{P}_{1}$ modulators, such as Gilenya ${ }^{\circledR}$ and ozanimod, which initially agonise $\mathrm{S}_{1} \mathrm{P}_{1}$ and then promote proteasomal degradation of the receptor (functional antagonism) to prevent T-cell egress and therefore limit deleterious effects of autoreactive T-cells on the central nervous system (CNS). Side effects of certain S1P receptor modulators include transient bradycardia, QT prolongation, macular edema, early reduced FEV, headache, anaemia, higher transaminase levels (liver), nasopharyngitis,

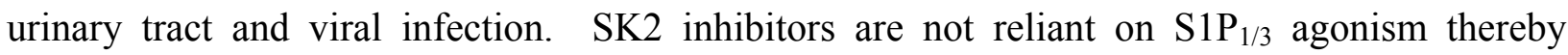
potentially minimising some of these side effects. Moreover, Yeliva ${ }^{\circledR}$ causes only a modest decrease in lymphocyte count. This is important, as a severe decrease in lymphocytes number may make patients susceptible to certain infections including rare, but fatal progressive multifocal leukoencephalopathy (PML). Indeed, lymphopenia is linked with reactivation of the JC virus, which causes PML [46]. The modest effect of Yeliva ${ }^{\circledR}$ on lymphocyte recirculation is supported by a partial or no effect on lymphopenia in Sphk2 $2^{-/}$mice $[24,47]$. Therefore, patients treated with SK2 inhibitors might have reduced risk from infection compared with the

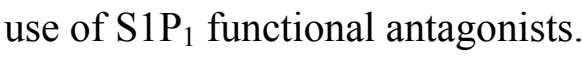

\section{Selectivity of SK2 inhibitors over SK1}

SK2-selective inhibitors reported to date include compounds 1-5 (Figure. 4, [26, 48-50]). Of these, Yeliva ${ }^{\circledR}$ (1) is the most advanced and is currently in Phase $1 / 2$ clinical trials for oncological indications. However, currently available SK2 inhibitors exhibit modest potency and/or selectivity over SK1 and, in some cases, modulate other targets. Thus, Yeliva ${ }^{\circledR}$ exhibits SK2-inhibitory potency in the micromolar range, inhibits dihydroceramide desaturase and 
induces proteasomal degradation of SK1 in cancer cells $[51,52]$. A key consideration, then, is the feasibility of developing compounds that combine high potency with high selectivity to facilitate exploration of the full potential of SK2 as a pharmacological target for clinical intervention.

Currently no SK2 crystal structures are available, but sequence comparison reveals strong homology to SK1, for which co-crystal structures with sphingosine (Sph) and with three Sphcompetitive inhibitors (6-8, Figure 4) have recently been solved [53-55]. The enzyme has a bidomain structure with a Sph-binding C-terminal domain (CTD) and an ATP-binding Nterminal domain (Figure 5A/B). The CTD adopts a two-layer $\beta$-sandwich with three 'lipid binding loops' (LBL-1 to LBL-3) folding across one face to generate the Sph-binding cavity, termed the 'J-channel'. A fourth inter-strand loop (the R-loop) packs on the opposite face of the CTD $\beta$-sandwich and fulfills a regulatory role. This loop, which is much extended in SK2 and hosts the phosphorylation-dependent nuclear export signal sequence, constitutes one of the key differences between SK2 and SK1. Of the three lipid binding loops, LBL-1 consists of two reverse-paired helices $(\alpha 7 / \alpha 8)$ and may gate access to the J-channel by a hinged motion [56]. Residues in LBL-1 and LBL-2 contribute to the contact surface for the polar head group of the lipid and central region. LBL-3, which contains an additional helix ( $\alpha 9)$ running antiparallel along $\alpha 8$, encloses much of the lipid tail.

Of the ca. 20 residues contributing to the ligand contact surface of the J-channel, SK2 differs from SK1 in only three; these are Ile174, Met272 and Phe288 of SK1, corresponding respectively to Val304, Leu517 and Cys533 in SK2. Ile174 and Met272 contribute to the stem of the J-channel. In the SK1 co-crystal structure with PF-543 (8) (Figure 5C), these residues flank the inhibitor's $p$-xylylene subunit; their replacement by Val and Leu may result in some recontouring of SK2 J-channel surface in this region. Phe288 plugs the toe of the SK1 J-channel; the cognate residue (Cys533) in SK2 is likely to result in a longer J-channel. However, 
homology modelling suggests that isoform differences in non-contact residues may subtly adjust packing of LBLs and potentially impact on the conserved heel region of the J-channel, leading to some compaction in SK2 (Figure 5D).

Another non-contact residue difference that may have bearing on development of SK2selective inhibitors is the substitution of Ala339 in SK1 by Thr584 in SK2. This residue lies proximal to a conserved aspartate (Asp178 in SK1) that engages the polar head group of Sph and inhibitors such as PF-543. Homology models indicate that presentation of the cognate aspartate (Asp308) in SK2 may be rotationally constrained by a hydrogen bond to Thr584 (Figure 5E). This, in turn, may impact on the optimal binding position of a basic centre within a ligand head group. In the case of the hydroxymethylpyrrolidine subunit of PF-543, this would suggest a preference for a slightly more deeply seated ammonium centre when bound to SK2. The pronounced SK1-over-SK2 inhibitory selectivity of PF-543 may then result from a tendency for a modest backward shunt of the ligand tail to sub-optimally compress the sulfonylmethylene linker in the heel region of the J-channel, which we suggest is tighter in SK2 than SK1 due to compaction of LBL-3. Together these considerations suggest a strategy for the design of improved SK2 inhibitors by optimizing the ligand tail with softened steric demand in the heel and increased steric demand in the toe relative to SK1-selective inhibitors. Combining such a tail with a head group that doubly engages Asp308 (via OD1/OD2 oxygen centres) and spacer subunits of optimal length to bridge between the tail and head group may provide access to potent SK2 inhibitors with enhanced selectivity over SK1.

Finally, given the flap-like structure of the lipid binding loops, we should also consider protein flexibility and whether 'tunneling' of inhibitor tail subunits might be possible either between LBL-1/3 or between LBL-2/3. Thus, Schnute et al. [50] have recently disclosed Pfizer27c (5) as a potent SK2 inhibitor with modest selectivity over SK1. These authors note that direct mapping of Pfizer-27c onto SK1-bound PF-543 reveals that the terminal oxadiazole ring is 
too large for accommodation in the heel of the J-channel, and thus it is proposed that phenoxy ring is rotated to present the oxazdiazole between helices $\alpha 8$ and $\alpha 9$ of LBL-1 and LBL-3. Validation of such a binding mode might open alternative strategies for optimizing SK2selective inhibitors to take advantage of isoform differences in residues not directly contributing to the J-channel.

\section{Concluding Remarks}

One has to carefully de-convolute data obtained with Sphk $2^{-/-}$mice to enable better definition of the role of SK2 in autoimmune/inflammatory disease and to provide a rationale for targeting this enzyme. This also raises a number of questions and areas for future research (see Outstanding Questions). Moreover, more highly potent and selective SK2 isoform inhibitors are needed to validate the role of SK2 and to allow optimisation and translation to the clinic. Such inhibitors can be used to establish whether the regulation of IL-12-stimulated IFN- $\gamma$ formation by SK2 is major determinant in promoting autoimmune disease, using, for instance, the IL- $10^{-/-}$mouse model of Crohn's disease. Such inhibitors can also be used to assess whether they reduce HDAC-1 levels and block Th17 polarisation in, for instance, EAE with comparison with Sphk2 ${ }^{-/-}$ mice models. Therefore, further studies on the role of SK2 in autoimmune/inflammatory disease are required in the future.

\section{References}

1. Pyne, S. and Pyne, N.J. (2011) Translational aspects of sphingosine 1-phosphate biology. Trends Mol. Med. 17, 463-472.

2. Chun, J. et al. (2002) International Union of Pharmacology. XXXIV. Lysophospholipid receptor nomenclature. Pharmacol. Rev. 54, 265-269. 
3. Kihara, Y. et al. (2014) Lysophospholipid receptor nomenclature review: IUPHAR Review 8. Br. J. Pharmacol. 171, 3575-3594.

4. Hait, N.C., et al. (2009) Regulation of histone acetylation in the nucleus by sphingosine-1phosphate. Science $325,1254-7$.

5. Panneer Selvam, S. et al. (2015) Binding of the sphingolipid S1P to hTERT stabilizes telomerase at the nuclear periphery by allosterically mimicking protein phosphorylation. Sci. Signal. 8:ra58.

6. Strub, G.M. et al. (2011) Sphingosine-1-phosphate produced by sphingosine kinase 2 in mitochondria interacts with prohibitin 2 to regulate complex IV assembly and respiration. FASEB J. 25, 600-612.

7. Mizugishi, K. et al. (2005) Essential role for sphingosine kinases in neural and vascular development. Mol. Cell Biol. 25, 11113-11121.

8. Hannun, Y.A. and Obeid, L.M. (2008) Principles of bioactive lipid signalling: lessons from sphingolipids. Nat. Rev. Mol. Cell Biol. 9, 139-150.

9. Pitson, S.M. et al. (2003) Activation of sphingosine kinase 1 by ERK1/2-mediated phosphorylation. EMBO J. 22, 5491-5500.

10. Hait, N.C. et al. (2007). Sphingosine kinase type 2 activation by ERK-mediated phosphorylation. J. Biol. Chem. 282, 12058-12065.

11. Maceyka, M. et al. (2005) SphK1 and SphK2, sphingosine kinase isoenzymes with opposing functions in sphingolipid metabolism. J. Biol. Chem. 280, 37118-37129.

12. Ding, G. et al. (2007) Protein kinase D-mediated phosphorylation and nuclear export of sphingosine kinase 2. J. Biol. Chem. 282, 27493-27502.

13. Liang, J. et al. (2013) Sphingosine-1-phosphate links persistent STAT3 activation, chronic intestinal inflammation, and development of colitis-associated cancer. Cancer Cell. 23, $107-$ 120. 
14. Pyne, N.J. and Pyne, S. (2013) Sphingosine 1-phosphate is a missing link between chronic inflammation and colon cancer. Cancer Cell 23, 5-7.

15. Gao, P. and Smith, C.D. (2011) Ablation of sphingosine kinase-2 inhibits tumor cell proliferation and migration. Mol Cancer Res. 9, 1509-1519.

16. Nagahashi, M. et al. (2016) Sphingosine-1-phosphate in the lymphatic fluid determined by novel methods. Heliyon. 2(12):e00219.

17. Snider, A.J. et al. (2009) A role for sphingosine kinase 1 in dextran sulfate sodium-induced colitis. FASEB J. 23,143-152.

18. Kharel, Y. et al. (2011) Sphingosine kinase type 2 inhibition elevates circulating sphingosine 1-phosphate. Biochem. J. 2012, 447, 149-157.

19. Beljanski, V. et al. (2011) Antitumor activity of sphingosine kinase 2 inhibitor ABC294640 and sorafenib in hepatocellular carcinoma xenografts. Cancer Biol Ther. 11, 524-34.

20. Samy, E.T. et al. (2007) Cutting edge: Modulation of intestinal autoimmunity and IL-2 signalling by sphingosine kinase 2 independent of sphingosine 1-phosphate. J. Immunol. $179,5644-5648$.

21. Bajwa A. et al. (2016) Sphingosine Kinase 2 Deficiency Attenuates Kidney Fibrosis via IFN- $\gamma$. J Am Soc Nephrol. doi: 10.1681/ASN.2016030306).

22. Jo, S.K. et al. (2009) Divergent roles of sphingosine kinases in kidney ischemia-reperfusion injury. Kidney Int. 75, 167-175

23. Jung, I.D. et al. (2007) Sphingosine kinase inhibitor suppresses a Th1 polarization via the inhibition of immunostimulatory activity in murine bone marrow-derived dendritic cells. Int. Immunol. 19, 411-426. 
24. Imeri, F. et al. (2016) Sphingosine kinase 2 deficient mice exhibit reduced experimental autoimmune encephalomyelitis: Resistance to FTY720 but not ST-968 treatments. Neuropharmacology 105, 341-350.

25. Barbour, M. et al. (2017) Effect of sphingosine kinase modulators on interleukin-1 $\beta$ release, sphingosine 1-phosphate receptor 1 expression and experimental autoimmune encephalomyelitis.Br. J. Pharmacol. 174, 210-222.

26. French, K.J. et al. (2010) Pharmacology and antitumor activity of ABC294640, a selective inhibitor of sphingosine kinase-2. J. Pharmacol. Exp. Ther. 333, 129-139.

27. Maines, L.W. et al. (2010) Efficacy of a novel sphingosine kinase inhibitor in experimental Crohn's diseaseInflammopharmacol. 18, 73-75.

28. Maines, L.W. et al. (2008) Suppression of ulcerative colitis in mice by orally available inhibitors of sphingosine kinase. Dig Dis Sci. 53, 997-1012.

29. Fitzpatrick, L.R. et al. (2011) Attenuation of arthritis in rodents by a novel orally-available inhibitor of sphingosine kinase. Inflammopharmacology 19, 75-87.

30. Xi, M. et al. (2016) Development of hydroxy-based sphingosine kinase inhibitors and antiinflammation in dextran sodium sulfate induced colitis in mice. Bioorg. Med. Chem. 24, $3218-3230$.

31. Newton, J. et al. (2015) Revisiting the sphingolipid rheostat: Evolving concepts in cancer therapy. Exp. Cell Res. 333, 195-200.

32. Brinkmann, V. et al. (2004) FTY720: sphingosine 1-phosphate receptor-1 in the control of lymphocyte egress and endothelial barrier function. Am. J. Transplant. 4, 1019-1025.

33. Vaclavkova, A. et al. (2014) Oral ponesimod in patients with chronic plaque psoriasis: a randomised, double-blind, placebo-controlled phase 2 trial. Lancet 384, 2036-2045.

34. Brinkmann, V. (2009) FTY720 (fingolimod) in Multiple Sclerosis: therapeutic effects in the immune and the central nervous system. Br. J. Pharmacol. 158, 1173-1182. 
35. Yoshimoto, T. et al. (2003) Positive modulation of IL-12 signalling by sphingosine kinase 2 associating with the IL-12 receptor beta 1 cytoplasmic region. J. Immunol. 171, 1352-1359.

36. Watford, W.T. et al. (2004) Signalling by IL-12 and IL-23 and the immunoregulatory roles of STAT4. Immunol. Rev. 202, 139-156.

37. Liao, J.J. et al. (2007) Cutting edge: Alternative signalling of Th17 cell development by sphingosine 1-phosphate. J. Immunol. 178, 5425-5428.

38. Lee, H. et al. (2010) STAT3-induced S1PR1 expression is crucial for persistent STAT3 activation in tumors. Nat. Med. 16, 1421-1428.

39. Yang, X.O. et al. (2007) STAT3 regulates cytokine-mediated generation of inflammatory helper T cells. J. Biol. Chem. 282, 9358-63.

40. Ivanov, I.I. et al. (2006) The orphan nuclear receptor RORgammat directs the differentiation program of proinflammatory IL-17+ T helper cells. Cell 126, 1121-1133.

41. Liu, G. et al. (2010) The S1P(1)-mTOR axis directs the reciprocal differentiation of $\mathrm{T}(\mathrm{H}) 1$ and T(reg) cells. Nat. Immunol. 11, 1047-1056.

42. Pyne, N.J. et al. (2013) Role of sphingosine 1-phosphate and lysophosphatidic acid in fibrosis. Biochim. Biophys. Acta. 1831, 228-238.

43. Wu, Q. et al. (2015) Reciprocal regulation of ROR $\gamma t$ acetylation and function by $\mathrm{p} 300$ and HDAC1. Sci Rep. 5, 16355.

44. Hait, N.C. et al. (2009) Regulation of histone acetylation in the nucleus by sphingosine-1phosphate. Science 325, 1254-1257.

45. Purvis, H.A. et al. (2010) Low strength T-cell activation promotesTh17 responses. Blood $116,4829-4837$.

46. Van Assche, G. et al. (2005) Progressive multifocal leukoencephalopathy after natalizumab therapy for Crohn's disease. N. Eng. J. Med. 353, 362-368. 
47. Kharel, Y. et al. (2005) Sphingosine kinase 2 is required for modulation of lymphocyte traffic by FTY720. J. Biol. Chem. 280, 36865-36872.

48. Liu, K. et al. (2013) Biological characterization of 3-(2-aminoethyl)-5-[3-(4-butoxylphenyl)propylidene]thiazolidine-2,4-dione (K145) as a selective sphingosine kinase-2 inhibitor and anticancer agent. PLoS ONE 8, e56471.

49. Lim, K.G. et al. (2011) (R)-FTY720 methyl ether is a specific sphingosine kinase 2 inhibitor: Effect on sphingosine kinase 2 expression in HEK 293 cells and actin rearrangement and survival of MCF-7 breast cancer cells. Cell. Signal. 23, 1590-1595.

50. Schnute, M. E, et al. (2017) Discovery of a potent and selective sphingosine kinase 1 inhibitor through the molecular combination of chemotype distinct screening hits. J. Med. Chem. in press (DOI: 10.1021/acs.jmedchem.7b00070).

51. McNaughton, M. et al. (2016) Proteasomal degradation of sphingosine kinase 1 and inhibition of dihydroceramide desaturase by the sphingosine kinase inhibitors, SKi or ABC294640, induces growth arrest in androgen-independent LNCaP-AI prostate cancer cells. Oncotarget 2016, 7, 16663-16675.

52. Venant, H. et al. (2015) The sphingosine kinase 2 inhibitor ABC294640 reduces the growth of prostate cancer cells and results in accumulation of dihydroceramides in vitro and in vivo. Mol. Cancer Ther. 14, 2744-2752.

53. Gustin, D.J. et al. (2013) Structure guided design of a series of sphingosine kinase (SphK) inhibitors. Bioorg. Med. Chem. Lett. 23, 4608-4616.

54. Wang, J. et al. (2014) Crystal Structure of sphingosine kinase 1 with PF-543. ACS Med. Chem. Lett. 5, 1329-1333.

55. Wang, Z. et al. (2013) Molecular basis of sphingosine kinase 1 substrate recognition and catalysis. Structure 21, 798-809. 
56. Adams, D.R. et al. (2016) Sphingosine kinases: emerging structure-function insights. Trends Biochem. Sci. 41, 395-409.

57. French, K.J. et al. (2003) Discovery and evaluation of inhibitors of human sphingosine kinase. Cancer Res. 63, 5962-5969.

\section{Figure Legends}

Figure. 1 Effect of inhibiting SK2 on S1P-dependent autoimmune/inflammatory disease forming mechanisms. The schematic shows that inhibiting SK2 activity can potentially reduce the availability of S1P at S1P receptors, which regulates Th17 polarisation. In addition, S1P formed by SK2 can bind to intracellular targets such as HDAC-1/2, which can regulate ROR- $\gamma \mathrm{t}$ (a master transcriptional regulator of Th17 polarisation) stability and suggesting that SK2 inhibitors might therefore reduce ROR- $\gamma$ t levels.

Figure. 2 Role of SK2 in regulating IL-12-dependent IFN- $\gamma$ from Th1 cells. The schematic shows that inhibiting SK2 might reduce signal transmission from the IL-12 $\beta 1$ receptor, which has been shown to form a complex with SK2. Indeed, a dominant negative SK2 mutant has been shown to reduce IL-12-stimulated IFN- $\gamma$ formation.

Figure. 3 Potential for combination approaches using SK2 inhibitors with IL-12/IL-17 antibodies or ROR- $\gamma$ t inverse agonists. Possible strategies for use of potent SK2 inhibitors with selectivity over SK1 in combination with anti-IL-12 or anti-IL-17 antibodies or ROR- $\gamma$ t inverse agonists to target Th1/Th17-mediated autoimmune/inflammatory disease.

Figure. 4. Structure of representative SK inhibitors with inhibitory activity data. 
Figure. 5. Key Figureure. Structure of SK1 with SK2 homology annotations. (A) The structure of SK1 with bound PF-543 (8) is shown (PDB: 4V24). The position of the ATPbinding site is marked with ADP (pink surface), superimposed from the SK1 crystal structure (3VZD) with bound Mg-ADP. Three lipid binding loops (LBL-1 to LBL-3, colour coded as indicated in the ribbon key) pack to generate the Sph-binding J-channel (coloured mesh). (B) As panel (A) but with $90^{\circ}$ rotation about the horizontal axis to highlight regions of postulated compaction and potential tunneling relevant to the design of SK2 inhibitors. (C) Detail of the SK1 J-channel surface (grey mesh) extracted from (A) and highlighting three isoform differences in surface-contributory residues; bound PF-543 (green stick) is visible within the surface. (D) SK1 J-channel surface (grey mesh) with superimposed modelled SK2 J-channel surface (magenta), highlighting regions of postulated compression and expansion relative to SK1.

SK1 J-channel surface (grey mesh) with bound PF-543 (green stick) rotated to view residues relevant to ligand head group engagement (SK1 Asp178 and Ala339, yellow stick); cognate SK2 residues (Asp308 and Thr584, thin blue stick) are shown superimposed from a homology model, where Thr584 is predicted to engage and constrain presentation of Asp308 to impact on the optimal positioning of bound ligand. 


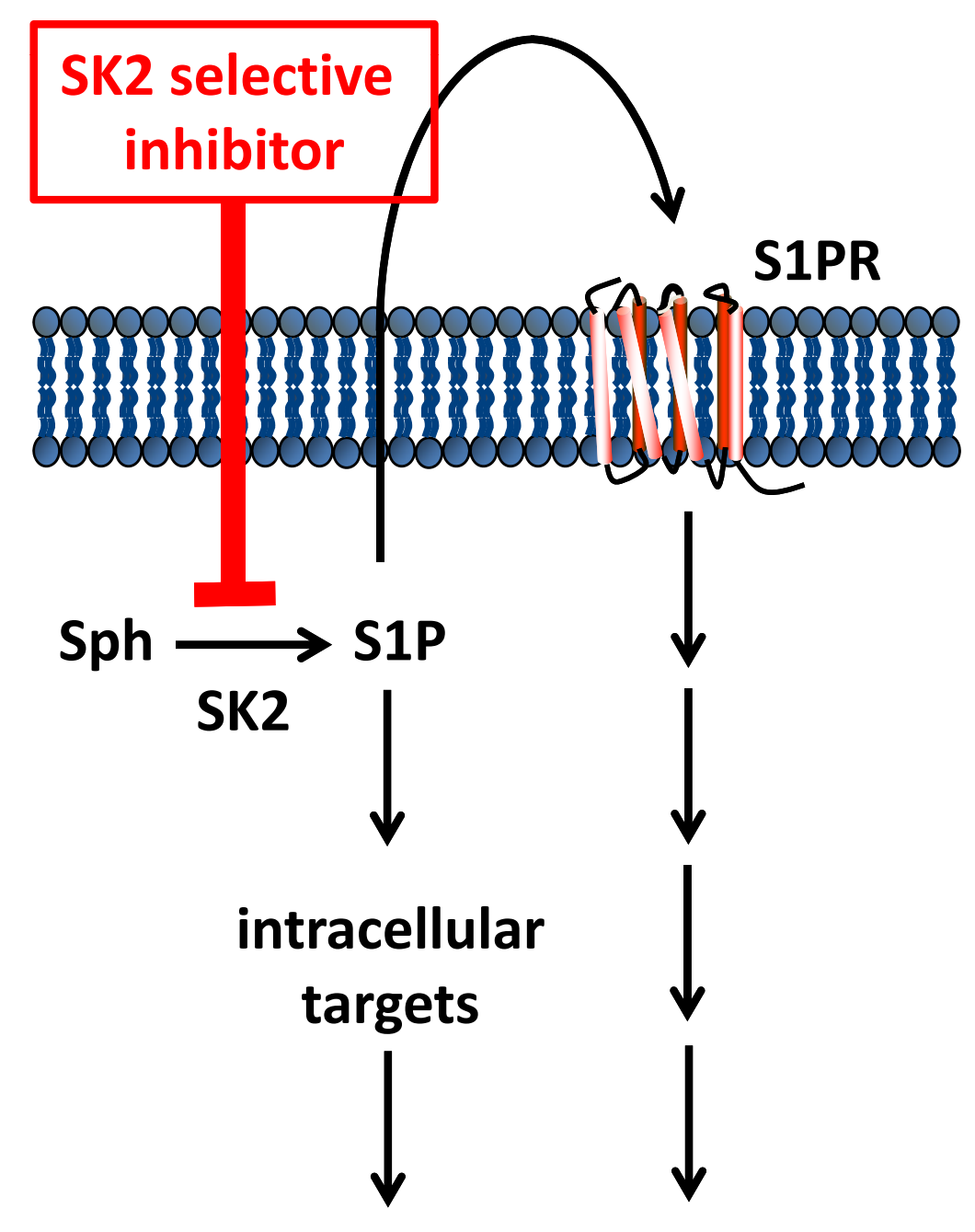

Innate/adaptive immune de-regulation

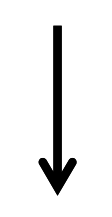

Auto-immune disease 


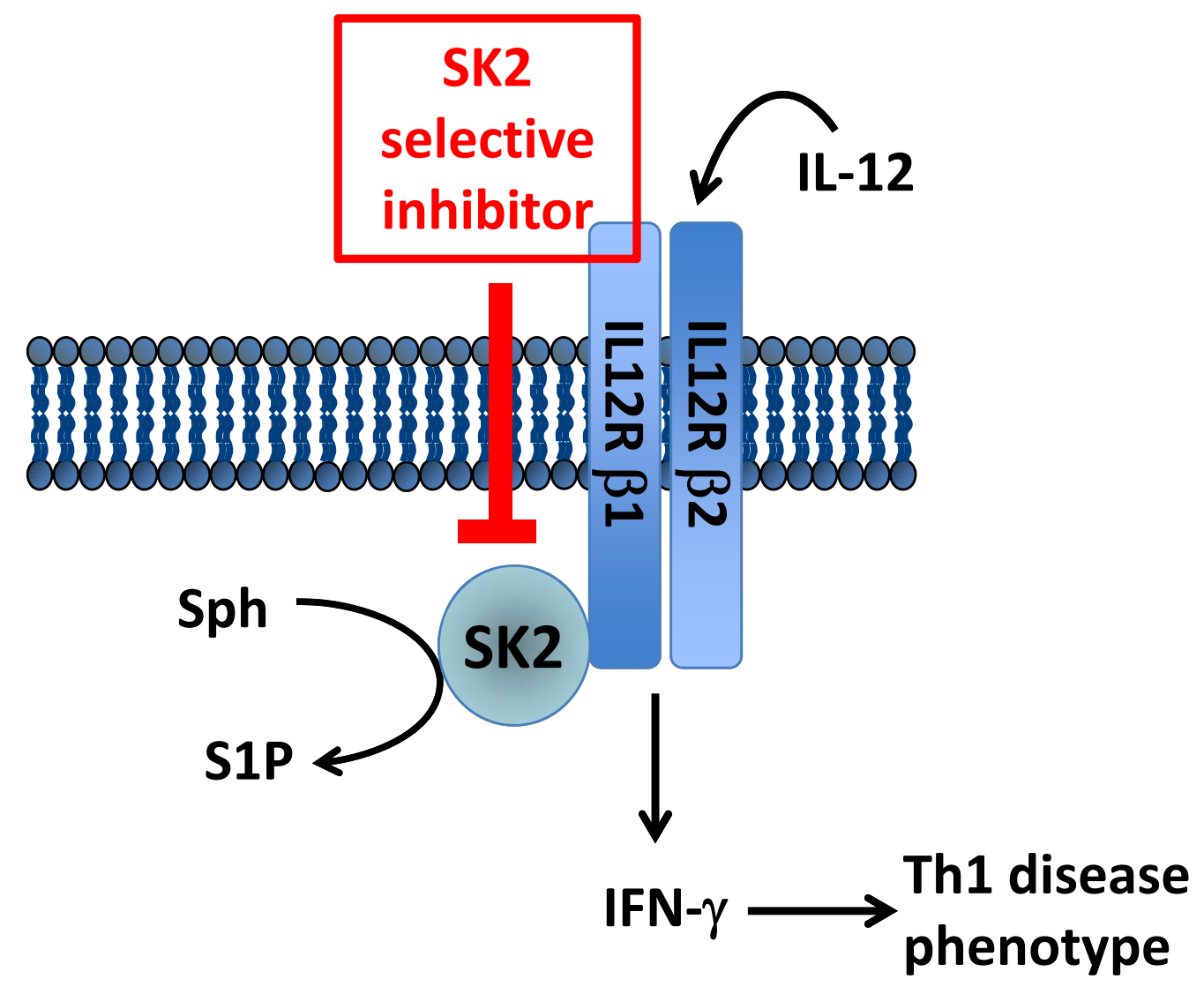




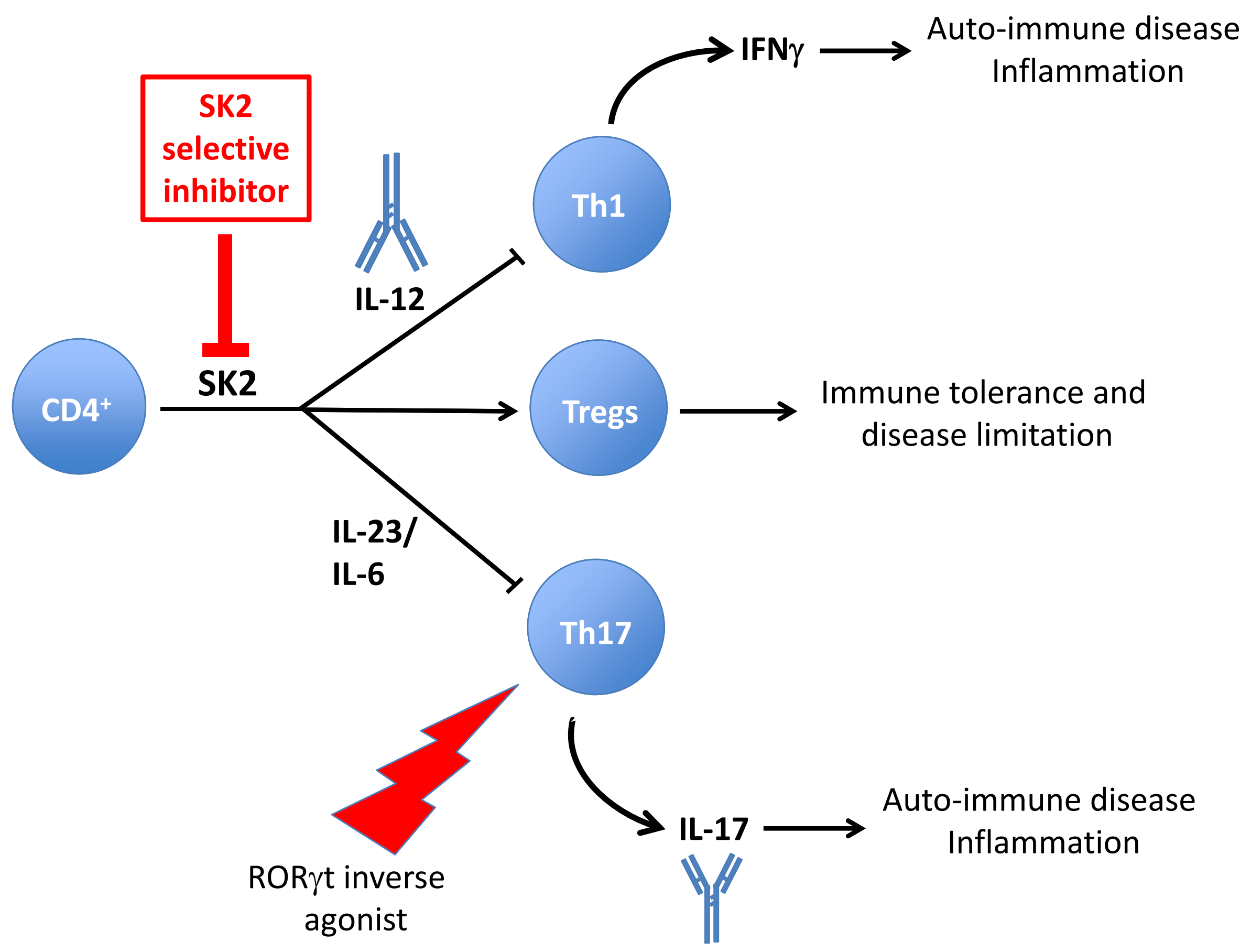


(1) $\mathrm{ABC} 294640$

(2) K145<smiles>CCCCOc1ccc(CC/C=C2\SC(=O)N(CCN)C2=O)cc1</smiles>

no inhibition $\quad 9,800$

(3) SLR080811<smiles>CCCCCCCCc1ccc(-c2noc(C3CCCN3C(=N)N)n2)cc1</smiles>

(4) ROMe

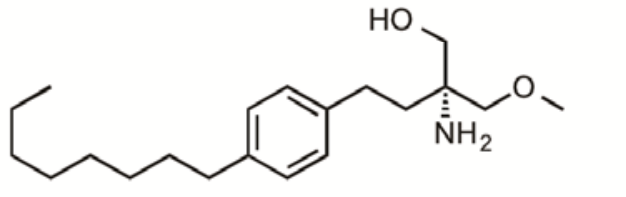

no inhibition 16,000 [49] at $100 \mu \mathrm{M}$

5) Pfizer-27c

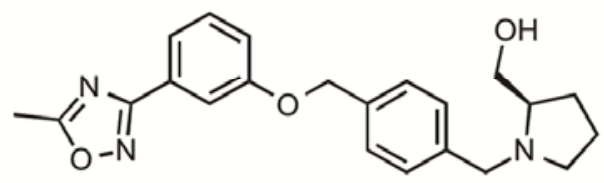

unselective

(6) SKi (П)

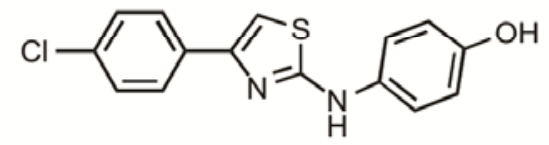

SK1-selective

(7) Amgen-82 (П)

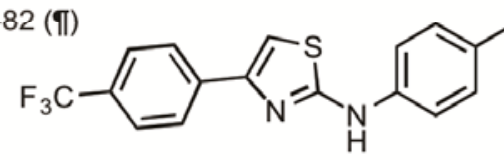

$\mathrm{HO}^{\mathrm{OH}}$

(114) [53]

(8) PF-543 (T)

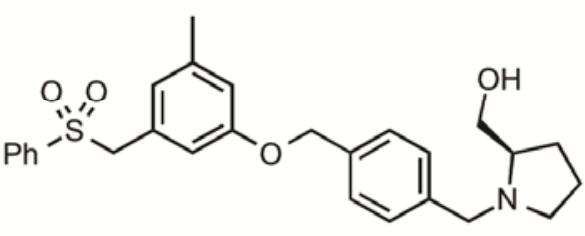


(A)

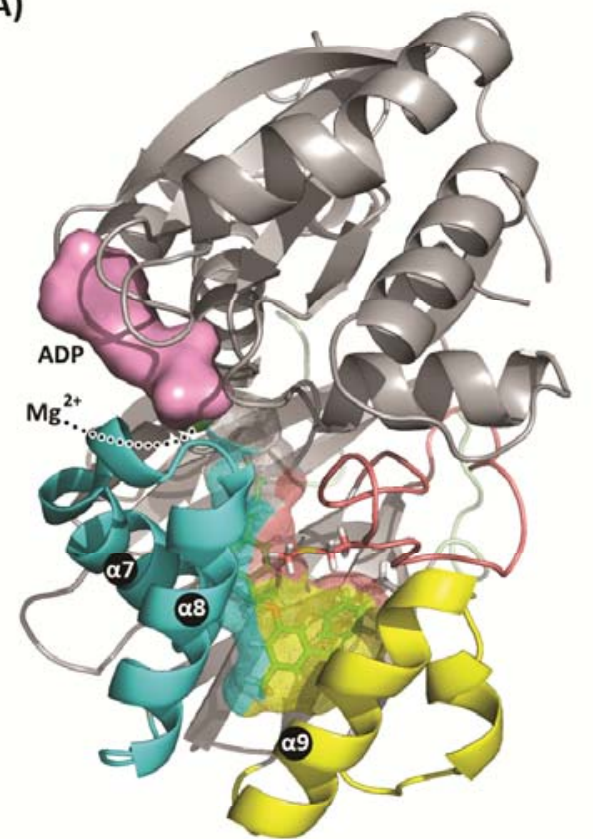

Ribbon key and SK1/SK2 homology index:

$$
\begin{array}{ll}
\text { LBL-1 } & \text { LBL-2 } \\
34 \text { residues } & 21 \text { residues } \\
62 \% \text { identity } & 43 \% \text { identity }
\end{array}
$$

(D)

(C)

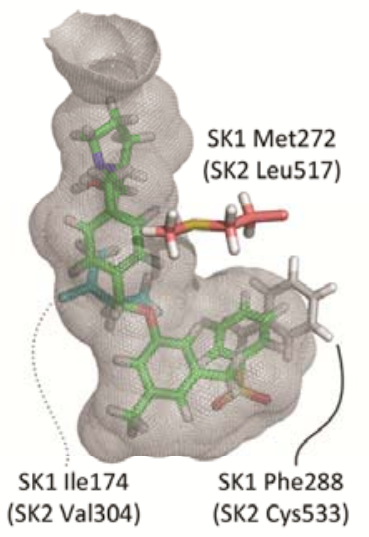

(B)

region of postulated surface compaction

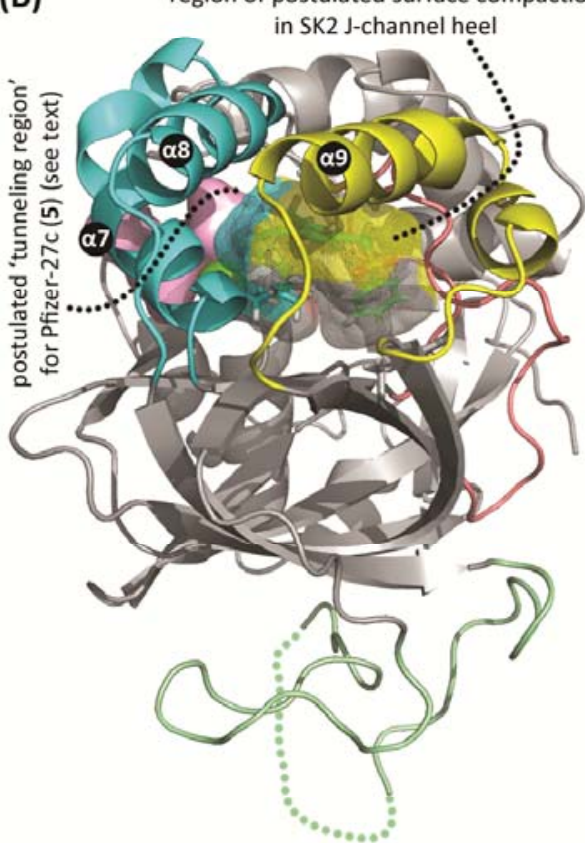

LBL-3
28 residues $61 \%$ identity

R-loop

SK1: 35 residues SK2: 151 residues

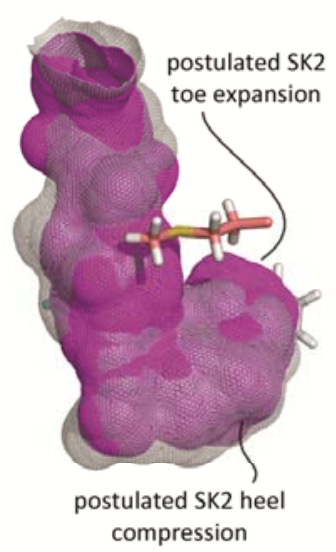

(E)

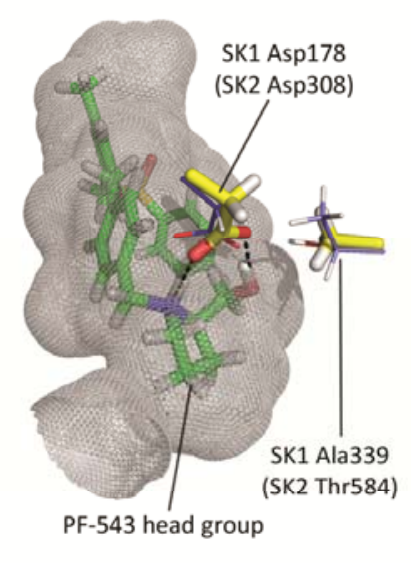

